\title{
LA EVOLUCIÓN DEL SALARIO EN UNA EMPRESA TEXTIL ALGODONERA. LA FÁBRICA DE LA RAMBLA DE VILANOVA I LA GELTRÚ (1891-1925)*
}

\author{
RAIMON SOLER I BECERRO \\ Universitat de Barcelona
}

\section{RESUMEN}

Esta nota es una primera aproximación a la evolución de los salarios en una empresa de tejidos de algodón catalana. A partir de la documentación de la misma empresa se ha elaborado un índice de salarios masculinos y otro de salarios femeninos. Las principales conclusiones que podemos extraer son la relativa estabilidad de los salarios hasta la I Guerra Mundial, el cambio en la evolución que se produjo a partir de ella, la diferenciación sexual del salario y la evolución diferente entre ambos indices.

\section{ABSTRACT}

This note is a first aproximation to the evolution of wages in a catalan cotton firm. From the firm sources it has been made a male and female wages indexes. The main conclusions are the relative wage stability up World War I, the change in the evolution from the war, the sexual diferentiation of wages and the different evolution between both indexes.

\section{INTRODUCCIÓN}

La atención hacia la evolución histórica de los salarios, especialmente en lo que hace referencia a su comportamiento desde los inicios de la Revolución Industrial, ha sido uno de los ejes principales que ha centrado numerosos

* El presente artículo tiene su origen en un trabajo realizado en la asignatura «Temes avançats en Història Econòmica» impartida por Albert Carreras en el marco del Programa Interuniversitari de Doctorat en Història Econòmica que realizan el Departament d'Història i Institucions Econò miques y el Departament d'Economia i Història Econòmica de las correspondientes facultades de ciencias económicas de la Universitat de Barcelona y de la Universitat Autònoma de Barcelona. El autor cuenta con una ayuda de la Fundación Caja de Madrid para la realización de su tesis doc. toral, la cual se centrará en el análisis integral de la evolución de la empresa objeto de esta nota. 
debates. Desde la preocupación por el nivel de vida de los trabajadores hasta el debate sobre el desarrollo y la distribución de la renta, son múltiples los campos de investigación en que el salario desempeña un papel fundamental. A pesar de que en España la preocupación por la evolución de los salarios es más reciente, las aportaciones en este campo se han multiplicado en los últimos años, aunque a menudo se han centrado en casos de una sola empresa, en un solo sector o se han limitado a un corto espacio temporal, hecho que se origina por la falta de estadísticas de carácter global. Por lo tanto los investigadores se han visto obligados a utilizar primordialmente las fuentes patrimoniales (ya sean de empresa, en el caso del sector industrial, o de propietarios, en el caso de la agricultura) ${ }^{1}$. La presente nota pretende hacer una aportación más al conocimiento de las características de la evolución de los salarios en la industria textil. Se trata de una primera aproximación al estudio de una empresa algodonera de tamaño medio. En un primer apartado se describe cómo se ha construido el índice, pasando después a plantearse cuál habría sido la evolución de los salarios en dicha empresa estableciendo términos de comparación que permitan considerar como mínimamente homologables los resultados obtenidos; finalmente se compara la evolución del salario industrial y agrario de la zona en que se sitúa la empresa: la comarca del Penedès.

La Fábrica de la Rambla se fundó en el año 1833 pero, por vicisitudes diversas, no pudo iniciar sus trabajos hasta mayo de $1839^{2}$. Esta fue la primera empresa de hilados y tejidos de algodón que funcionó en Vilanova i la Geltrú y, si bien fue una de las primeras fábricas catalanas en utilizar el vapor como energía, se convirtió rápidamente en una empresa de carácter mediano - tanto a nivel catalán como de la propia Vilanova - a medida que el proceso de industrialización fue avanzando ${ }^{3}$. La empresa abarcaba desde su fundación todos los ramos de la fabricación, desde el hilado hasta el tinte. En el período que comprende la presente nota fueron tres las razones sociales que se ocuparon de la fábrica, aunque la empresa utilizaba la marca «El Fé-

${ }^{1}$ Aunque son numerosos los trabajos realizados en toda Europa, un resumen de lo que es la bibliografía sobre el tema y una recopilación de varios trabajos se pueden consultar en Scholliers (1989). También se puede consultar el pequeño volumen recopilatorio editado por Vera Zamagni y el propio Scholliers en el marco del XI Congreso de Internacional de Historia Económica que recoge diversas comunicaciones del conjunto de Europa, incluyendo una de James Simpson referida a España. En el caso español son varios los trabajos publicados, pero creo que podemos destacar el de Maluquer de Motes (1989), las diversas aportaciones del Simposi d'Anàlisi Econòmica. Secció d'Història Econòmica (1990), el de Garrabou, Pujol y Colomé (1991) y el de Reher y Ballesteros (1993).

2 Sobre las vicisitudes de esta empresa hasta 1862 se puede consultar Nadal (1992).

${ }^{3}$ Por ejemplo, la fábrica era, en 1913, la tercera de cinco según la cotización en la matrícula industrial de Vilanova. Daba trabajo a una media de doscientas cincuenta personas y contaba con un capital de un millón de pesetas. 
nix» desde 1882. La primera razón social, Santacana y Cía., condujo la empresa entre 1889 y 1902; después, durante un corto espacio de dos años, pasó a denominarse Sadurni y Cía.; finalmente, el año 1904 se produjo un nuevo cambio de gerencia y la sociedad tomó el nombre de Alegre, Ferrer y Cía.

\section{LA CONSTRUCCIÓN DEL ÍNDICE}

En esta sección se describe cómo se han construido las series de salarios masculinos y femeninos y el índice general del salario nominal en esta industria textil y su correspondiente deflación.

La serie que presento se ha construido a partir de la información extraida de los Libros semanales de salarios correspondientes al Archivo de la Fábrica de la Rambla que se encuentra depositado en el Museu RomànticCan Papiol de Vilanova i la Geltrú ${ }^{4}$.

Los dieciocho volúmenes que existen abarcan el período 1891-1928. Solamente falta el correspondiente al año 1895 , pero he dado término a la serie en 1925 porque los últimos años presentan deficiencias que impedian darle coherencia. De los varios oficios que componían la escala laboral de la fábrica se ha elaborado una media aritmética de tres semanas por cada año; éstas son siempre las mismas y corresponden a los meses de febrero, junio y noviembre. Una vez hecho esto, se han agrupado los diversos oficios por secciones, siguiendo la estructura laboral de la fábrica y diferenciando por sexos. Para cada sección se ha elaborado una media aritmética con los oficios que comprendía. Las agrupaciones resultantes son: portería (formada por la categoría de los porteros y serenos), técnicos (trabajadores del motor, mecánicos, untador o electricista y carpinteros), preparación de hilados (batán, cardas, canal y mayordomo), en una cuarta sección se han incluido los salarios de la sección de aparar y, finalmente, se ha considerado también el sueldo de los auxiliares de continuas. Para los trabajos femeninos, las secciones en que se han dividido son: preparación de hilados (manuares, mecheras y ayudantas), hilados (trabajadoras de las continuas), una sección que incluye el salario de las urdidoras y nudadoras y, finalmente, la de las tejedoras. Así pues, el salario nominal resultante -que se muestra en el cuadro 1 - es una media aritmética de los salarios de las diversas secciones masculinas y femeninas, de la misma forma que el salario nominal de la fábrica es una media entre estas dos series, a partir de la cual se ha elaborado el índice del salario nominal. Cabe suponer que el resultado sería representativo del salario medio en la industria textil en la población de Vilanova i la Geltrú.

4 Quiero agradecer a Assumpta Gou y a Carmen Barroso, directora y bibliotecaria, respectivamente, de dicho museo, haber facilitado y permitido la consulta del material. 


\section{CUADRO 1}

A. Salarios nominales en las secciones de trabajos masculinas (pesetas corrientes)

\begin{tabular}{|c|c|c|c|c|c|c|c|}
\hline & Porteria & Técnicos & $\begin{array}{c}\text { Prep. } \\
\text { de } \\
\text { bilados }\end{array}$ & $\begin{array}{c}\text { Aspeo } \\
y \\
\text { blanqueo }\end{array}$ & Aparar & $\begin{array}{l}\text { Auxiliar } \\
\text { continua }\end{array}$ & $\begin{array}{c}\text { Salario } \\
\text { nominal } \\
\text { masculino }\end{array}$ \\
\hline 1891. & 15,25 & 26,66 & 17,16 & 13,11 & 28,00 & 20,00 & 20,03 \\
\hline $1892 \ldots \ldots \ldots$ & 15,25 & 26,26 & 17,16 & 14,16 & 28,00 & 20,00 & 20,14 \\
\hline $1893 \ldots \ldots \ldots$ & 15,25 & 26,30 & 17,16 & 15,95 & 28,00 & 20,00 & 20,44 \\
\hline $1894 \ldots \ldots \ldots$ & 15,25 & 24,90 & 14,66 & 16,20 & 28,00 & 20,00 & 19,84 \\
\hline $1895 \ldots \ldots \ldots$ & - & - & - & - & - & - & - \\
\hline $1896 \ldots \ldots \ldots$ & 15,75 & 22,90 & 16,58 & 14,71 & 23,00 & 25,00 & 19,66 \\
\hline $1897 \ldots \ldots \ldots$ & 15,75 & 20,84 & 16,58 & 15,13 & 28,00 & 25,00 & 20,22 \\
\hline $1898 \ldots \ldots \ldots$ & 15,75 & 25,37 & 16,58 & 15,45 & 20,50 & 25,00 & 19,78 \\
\hline $1899 \therefore \ldots \ldots$ & 15,75 & 25,37 & 16,58 & 15,22 & 20,50 & 25,00 & 19,74 \\
\hline $1900 \ldots \ldots . .$. & 15,75 & 20,90 & 16,70 & 16,65 & 20,50 & 25,00 & 19,25 \\
\hline $1901 \ldots \ldots . . .$. & 15,75 & 23,62 & 12,37 & 19,34 & 20,50 & 25,00 & 19,43 \\
\hline $1902 \ldots \ldots . . .$. & 15,75 & 23,90 & 13,83 & 24,38 & 20,50 & 25,00 & 20,56 \\
\hline $1903 \ldots \ldots . . .$. & 15,75 & 24,39 & 13,75 & 18,00 & 25,50 & 25,00 & 20,40 \\
\hline $1904 \ldots \ldots \ldots$ & 15,83 & 23,70 & 15,91 & 22,21 & 25,50 & 25,00 & 21,36 \\
\hline $1905 \ldots \ldots \ldots$ & 15,75 & 24,43 & 16,54 & 23,41 & 21,75 & 25,00 & 21,15 \\
\hline $1906 \ldots \ldots \ldots$ & 15,75 & 24,43 & 17,17 & 23,43 & 19,25 & 25,00 & 20,84 \\
\hline $1907 \ldots \ldots . . .$. & 15,75 & 24,48 & 16,61 & 23,85 & 19,25 & 25,00 & 20,82 \\
\hline $1908 \ldots \ldots \ldots$ & 15,75 & 24,23 & 16,67 & 24,56 & 19,25 & 25,00 & 20,91 \\
\hline $1909 \ldots \ldots \ldots$ & 15,75 & 23,60 & 16,50 & 24,90 & 19,25 & 25,00 & 20,83 \\
\hline $1910 \ldots \ldots \ldots$ & 15,75 & 24,97 & 16,50 & 27,58 & 19,25 & 25,00 & 21,51 \\
\hline $1911 \ldots \ldots \ldots$ & 15,92 & 30,50 & 16,50 & 26,53 & 19,25 & 25,00 & 22,28 \\
\hline $1912 \ldots \ldots . . .$. & 16,00 & 32,25 & 13,86 & 21,66 & 14,75 & 25,00 & 20,59 \\
\hline $1913 \ldots \ldots \ldots$ & 16,00 & 28,00 & 14,37 & 16,79 & 19,33 & 23,33 & 19,64 \\
\hline $1914 \ldots \ldots \ldots$ & 16,00 & 26,56 & 15,93 & 20,06 & 19,67 & 23,33 & 20,26 \\
\hline $1915 \ldots \ldots \ldots$ & 16,00 & 22,71 & 15,45 & 19,90 & 20,50 & 25,00 & 19,93 \\
\hline $1916 \ldots \ldots . . . . .$. & 16,00 & 22,87 & 16,07 & 22,86 & 20,50 & 25,00 & 20,55 \\
\hline $1917 \ldots \ldots \ldots$ & 16,17 & 23,74 & 16,63 & 22,93 & 25,00 & 25,00 & 21,58 \\
\hline $1918 \ldots \ldots \ldots$ & 16,50 & 26,83 & 15,73 & 22,29 & 25,00 & 25,00 & 21,89 \\
\hline $1919 \ldots \ldots \ldots$ & 19,33 & 33,66 & 23,21 & 28,69 & 31,67 & 27,50 & 27,34 \\
\hline $1920 \ldots \ldots \ldots$ & 32,08 & 54,33 & 31,96 & 45,66 & 43,35 & 28,33 & 39,28 \\
\hline $1921 \ldots \ldots \ldots$ & 42,50 & 60,67 & 38,86 & 55,44 & 53,34 & 30,00 & 46,80 \\
\hline $1922 \ldots \ldots \ldots$ & 42,50 & 76,67 & 41,52 & 64,46 & 57,17 & 34,17 & 52,75 \\
\hline $1923 \ldots \ldots \ldots$ & 42,50 & 65,26 & 42,76 & 62,59 & 56,68 & 30,00 & 49,96 \\
\hline $1924 \ldots \ldots \ldots$ & 42,50 & 59,31 & 37,32 & 53,53 & 55,73 & 30,00 & 46,40 \\
\hline $1925 \ldots \ldots \ldots$ & 42,50 & 59,10 & 36,29 & 53,16 & 55,73 & 30,00 & 46,13 \\
\hline
\end{tabular}




\section{CUADRO 1 (continuación)}

B. Salarios nominales en las secciones de trabajos femeninas (pesetas corrientes)

\begin{tabular}{|c|c|c|c|c|c|}
\hline & $\begin{array}{c}\text { Prep. } \\
\text { de } \\
\text { bilados }\end{array}$ & $\begin{array}{c}\text { Hilatura } \\
\text { (cont.) }\end{array}$ & $\begin{array}{c}\text { Urdidoras } \\
y \\
\text { nudadoras }\end{array}$ & Telares & $\begin{array}{c}\text { Salario } \\
\text { nominal } \\
\text { femenino }\end{array}$ \\
\hline $1891 \ldots \ldots \ldots . . .$. & 10,54 & 10,50 & 12,70 & 8,00 & 10,43 \\
\hline $1892 \ldots \ldots . . .$. & 10,75 & 11,65 & 18,86 & 9,53 & 13,75 \\
\hline $1893 \ldots \ldots \ldots$ & 10,75 & 11,65 & 17,91 & 9,51 & 13,44 \\
\hline $1894 \ldots . . . . . . . .$. & 10,75 & 10,75 & 17,55 & 11,33 & 13,02 \\
\hline $1895 \ldots \ldots \ldots$ & - & - & - & - & - \\
\hline $1896 \ldots$ & 10,58 & 13,00 & 15,19 & 10,01 & 12,92 \\
\hline $1897 \ldots . . . . . .$. & 10,50 & 13,00 & 18,18 & 10,53 & 13,89 \\
\hline $1898 \ldots \ldots . . . . .$. & 10,50 & 13,00 & 12,92 & 10,84 & 12,14 \\
\hline $1899 \ldots . . . . . . .$. & 10,50 & 13,00 & 20,77 & 13,86 & 14,76 \\
\hline $1900 \ldots \ldots \ldots$ & 10,50 & 13,00 & 17,56 & 8,31 & 13,69 \\
\hline $1901 \ldots \ldots \ldots$ & 10,50 & 13,00 & 16,35 & 8,84 & 13,28 \\
\hline $1902 \ldots \ldots . . .$. & 10,50 & 13,00 & 23,33 & 9,58 & 15,61 \\
\hline $1903 \ldots \ldots . .$. & 10,50 & 13,00 & 18,99 & 9,35 & 14,16 \\
\hline $1904 \ldots . . . . . . . . .$. & 10,50 & 12,43 & 19,05 & 9,80 & 13,99 \\
\hline $1905 \ldots \ldots \ldots$ & 10,50 & 9,07 & 11,98 & 10,73 & 10,52 \\
\hline $1906 \ldots$ & 10,50 & 14,20 & 15,26 & 10,40 & 13,32 \\
\hline $1907 \ldots \ldots . . . .$. & 10,48 & 13,82 & 15,56 & 12,82 & 13,29 \\
\hline $1908 \ldots \ldots \ldots . .$. & 10,48 & 13,93 & 13,13 & 12,85 & 12,51 \\
\hline $1909 \ldots \ldots \ldots$ & 10,87 & 15,38 & 12,94 & 10,76 & 13,06 \\
\hline $1910 \ldots \ldots$ & 10,87 & 16,25 & 19,63 & 10,43 & 15,58 \\
\hline $1911 \ldots \ldots \ldots$ & 9,96 & 15,14 & 14,46 & 9,90 & 13,19 \\
\hline $1912 \ldots \ldots \ldots$ & 10,61 & 8,55 & 12,43 & 7,79 & 10,53 \\
\hline 1913 & 11,97 & 9,40 & 9,42 & 8,63 & 10,26 \\
\hline $1914 \ldots \ldots \ldots . .$. & 12,30 & 12,94 & 9,87 & 11,26 & 11,70 \\
\hline $1915 \ldots \ldots \ldots$ & 11,65 & 14,99 & 12,31 & 11,25 & 12,98 \\
\hline $1916 \ldots \ldots \ldots$ & 12,39 & 15,10 & 12,24 & 9,95 & 13,24 \\
\hline $1917 \ldots \ldots \ldots$ & 12,26 & 13,09 & 12,65 & 10,29 & 12,66 \\
\hline $1918 \ldots \ldots \ldots$ & 11,25 & 13,87 & 17,03 & 10,30 & 14,05 \\
\hline $1919 \ldots \ldots \ldots$ & 13,70 & 17,06 & 17,54 & 11,76 & 16,10 \\
\hline $1920 \ldots \ldots \ldots$ & 17,91 & 21,47 & 16,98 & 13,82 & 18,78 \\
\hline $1921 \ldots \ldots . . . .$. & 18,62 & 24,45 & 20,54 & 11,82 & 21,20 \\
\hline $1922 \ldots \ldots \ldots$ & 21,26 & 30,20 & 23,47 & 15,86 & 24,98 \\
\hline $1923 \ldots \ldots \ldots$ & 23,29 & 35,08 & 22,05 & 15,21 & 26,81 \\
\hline $1924 \ldots \ldots \ldots . . . . . . . .$. & 15,70 & 26,72 & 24,97 & 12,37 & 22,46 \\
\hline 1925 & 18,77 & 28,09 & 19,14 & 10,79 & 22,00 \\
\hline
\end{tabular}

FUENTE: Archivo de la Fábrica de la Rambla (AFR), Libros semanales de salarios (1890-1928). 
En cuanto al número de trabajadores que ocupaba la empresa, podemos señalar que se había ido reduciendo progresivamente. Así, según los datos que nos facilitan los mismos Libros semanales, en los años iniciales de la serie se habría dado ocupación a un total que oscilaría entre las 290 y 300 personas (teniendo en cuenta que no consideramos los trabajadores de las selfactinas y las variaciones que se producen de una semana a otra). Desde principios de siglo el número de personas ocupadas fue menor, especialmente a causa de la disminución de las secciones de telares y de aspeo. El cuadro 2 muestra el número de trabajadores que se ocuparon en cada una de las secciones consideradas en la serie. Para ello he utilizado el promedio de los trabajadores empleados en cada una de ellas en las tres semanas consideradas para construir la serie de salarios y en tres años diferentes. Como puede comprobarse, el porcentaje que representaron las mujeres sobre los hombres fue siempre más elevado, llegando, en determinados momentos, a significar casi un 70 por ciento de la mano de obra empleada.

\section{CUADRO 2}

Trabajadores empleados en las secciones consideradas para construir el indice de salarios de la Fábrica de la Rambla

\begin{tabular}{|c|c|c|c|c|c|c|}
\hline & \multicolumn{2}{|c|}{1896} & \multicolumn{2}{|c|}{1909} & \multicolumn{2}{|c|}{1923} \\
\hline & Trab. & $\%$ & Trab. & $\%$ & Trab. & $\%$ \\
\hline \multicolumn{7}{|l|}{ 1. Trabajos masculinos } \\
\hline 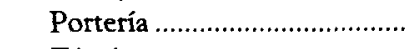 & 4 & 1,38 & 4 & 2,15 & 6 & 3,17 \\
\hline 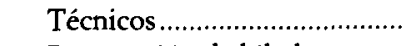 & 14 & 4,83 & 14 & 7,53 & 12 & 6,35 \\
\hline Preparaciớn de hilados ............ & 13 & 4,48 & 10 & 5,38 & 10 & 5,29 \\
\hline Aspeo y blanqueo ...................... & 56 & 19,31 & 18 & 9,68 & 27 & 14,29 \\
\hline 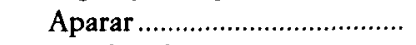 & 6 & 2,07 & 5 & 2,69 & 4 & 2,12 \\
\hline Auxiliar de continuas .............. & 2 & 0,69 & 6 & 3,23 & 6 & 3,17 \\
\hline 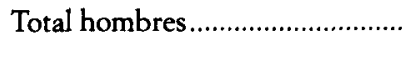 & 95 & 32,76 & 57 & 30,65 & 65 & 34,39 \\
\hline \multicolumn{7}{|l|}{ 2. Trabajos femeninos } \\
\hline Preparación de hilados........... & 47 & 16,21 & 39 & 20,97 & 40 & 21,16 \\
\hline 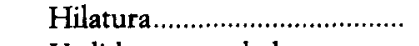 & 53 & 18,28 & 42 & 22,58 & 42 & 22,22 \\
\hline Urdidoras y nudadoras............ & 13 & 4,48 & 11 & 5,91 & 9 & 4,76 \\
\hline Telares ....................................... & 82 & 28,28 & 37 & 19,89 & 33 & 17,46 \\
\hline 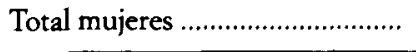 & 195 & 67,24 & 129 & 69,35 & 124 & 65,61 \\
\hline Total & 290 & 100,00 & 186 & 100,00 & 189 & 100,00 \\
\hline
\end{tabular}

FUENTE: La misma del cuadro 1. 
Como deflactor se ha tomado el índice del coste de la vida elaborado por Maluquer y que se encuentra publicado en las Estadísticas bistóricas de España ${ }^{5}$. Aunque no cubre la integridad del período, se trata de un índice que permite deflactar salarios sin tener que recurrir a un índice de precios al por mayor -hecho a veces inevitable, pero teóricamente incorrecto-, además de hacer referencia a Barcelona, una ciudad relativamente próxima a la población que se estudia y posiblemente con niveles de precios y de salarios, si no iguales, sí muy similares.

\section{SALARIO NOMINAL Y SALARIO REAL EN VILANOVA: LOS RESULTADOS}

Así pues, una vez obtenidos los resultados anteriormente expuestos, creo que podemos apuntar algunas tendencias respecto a cuál habría sido la evolución del salario. Hay que destacar el carácter relativamente estable que muestran los salarios nominales hasta 1917. Este hecho viene determinado por las pequeñas variaciones que presentaron los salarios nominales masculinos, mientras que el salario nominal femenino tiende a fluctuar más como consecuencia de un mayor porcentaje de trabajo a destajo. En este sentido, podemos remarcar que aunque los salarios masculinos se sitúan siempre, en términos monetarios, por encima de los femeninos, el índice de estos últimos tiende a crecer más que aquéllos. En cierto modo es lógico que fuese más fácil aumentar un salario bajo que uno situado a un nivel superior. Creo que aquí hay que tener en cuenta el proceso de feminización de la mano de obra que habría significado el cambio de las selfactinas por continuas. Este proceso se había iniciado a finales de los años setenta y se completó definitivamente a mediados de los años noventa del siglo pasado, hecho que habría permitido eliminar un coste laboral y unos salarios elevados como eran los de las selfactinas, mantener estables y rebajar, como consecuencia, los salarios de la preparación de hilados y de la hilatura y dejar los aumentos o las reducciones nominales de salarios concentrados en aquellos oficios cuyo salario se pagaba a destajo (esencialmente urdidoras y nudadoras y tejedoras), que son los que determinan las fluctuaciones. En general, como podemos ver, el índice de los salarios nominales muestra una cierta tendencia al crecimiento, aunque hay que destacar dos momentos de caida de los salarios en 1898 y en 1905, que podemos atribuir a la si-

s Maluquer (1989), p. 518. 


\section{CUADRO 3}

Salario nominal y salario real en Vilanova i la Geltrú (1891-1925)

\begin{tabular}{|c|c|c|c|c|c|c|}
\hline & (1) & (2) & (3) & (4) & (5) & (6) \\
\hline $1891 \ldots \ldots \ldots \ldots \ldots \ldots \ldots$ & 20,03 & 10,43 & 15,23 & 101,9 & & \\
\hline 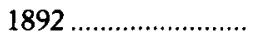 & 20,14 & 13,75 & 16,95 & 113,4 & & \\
\hline 1893 & 20,44 & 13,44 & 16,94 & 113,3 & & \\
\hline 1894 & 19,84 & 13,02 & 16,43 & 109,9 & & \\
\hline 1895 & - & - & - & - & & \\
\hline 1896 & 19,66 & 12,92 & 16,29 & 109,0 & & \\
\hline 1897 & 20,22 & 13,89 & 17,05 & 114,1 & & \\
\hline 1898 & 19,78 & 12,14 & 15,96 & 106,7 & & \\
\hline 1899 & 19,74 & 14,76 & 17,25 & 115,4 & & \\
\hline 1900 & 19,25 & 13,69 & 16,47 & 110,2 & & \\
\hline 1901 & 19,43 & 13,28 & 16,36 & 109,4 & & \\
\hline $1902 \ldots \ldots \ldots \ldots \ldots \ldots . .$. & 20,56 & 15,61 & 18,09 & 121,0 & & \\
\hline 1903 & 20,40 & 14,16 & 17,28 & 115,6 & & \\
\hline 1904 & 21,36 & 13,99 & 17,68 & 118,2 & & \\
\hline 1905 & 21,15 & 10,52 & 15,83 & 105,9 & & \\
\hline 1906 & 20,84 & 13,32 & 17,08 & 114,2 & & \\
\hline 1907 & 20,82 & 13,29 & 17,06 & 114,1 & & \\
\hline 1908 & 20,91 & 12,51 & 16,71 & 111,8 & & \\
\hline 1909 & 20,83 & 13,06 & 16,95 & 113,4 & 100,4 & 112,9 \\
\hline 1910 & 21,51 & 15,58 & 18,55 & 124,0 & 99,4 & 124,8 \\
\hline 1911 & 22,28 & 13,19 & 17,73 & 118,6 & 100,1 & 118,5 \\
\hline 1912 & 20,59 & 10,53 & 15,56 & 104,1 & 99,3 & 104,8 \\
\hline 1913 & 19,64 & 10,26 & 14,95 & 100,0 & 100,0 & 100,0 \\
\hline 1914 & 20,26 & 11,70 & 15,98 & 106,9 & 101,0 & 105,8 \\
\hline 1915 & 19,93 & 12,98 & 16,46 & 110,1 & 104,9 & 104,9 \\
\hline 1916 & 20,55 & 13,24 & 16,89 & 113,0 & 117,5 & 96,2 \\
\hline 1917 & 21,58 & 12,66 & 17,12 & 114,5 & 122,7 & 93,3 \\
\hline 1918 & 21,89 & 14,05 & 17,97 & 120,2 & 137,2 & 87,6 \\
\hline 1919 & 27,34 & 16,10 & 21,72 & 145,3 & 162,8 & 89,2 \\
\hline 1920 & 39,28 & 18,78 & 29,03 & 194,2 & 171,7 & 113,1 \\
\hline 1921 & 46,80 & 21,20 & 34,00 & 227,4 & 163,4 & 139,2 \\
\hline $1922 \ldots \ldots \ldots \ldots \ldots$ & 52,75 & 24,98 & 38,86 & 259,9 & 157,0 & 165,6 \\
\hline 1923 & 49,96 & 26,81 & 38,38 & 256,8 & 161,3 & 159,2 \\
\hline 1924 & 46,40 & 22,46 & 34,43 & 230,3 & 164,4 & 140,1 \\
\hline 1925 & 46,13 & 22,00 & 34,06 & 227,8 & 166,6 & 136,8 \\
\hline
\end{tabular}

NotaS: (1) Salario nominal masculino; (2) Salario nominal femenino; (3) Salario nominal medio de la Fábrica de la Rambla; (4) Índice del salario nominal, $1913=100$; (5) Índice del coste de la vida; (6) Salario real: (4)/(5).

FUENTE: Libros semanales de salarios (1890-1928). Indice de precios: Maluquer (1989), pp. 518-519. 
tuación crítica por la que pasó la empresa en aquellos años, mientras que la caída brusca de los salarios que se produce en 1912 es probable que la tengamos que atribuir a una reducción del número de telares que se produce en aquel año y a los cambios tecnológicos que se habrían comenzado a introducir en las primeras décadas del siglo, coincidiendo con el cambio de razón social en 1904: introducción de los telares de panas, electrificación del proceso productivo, etc. En resumen, podemos considerar, tal como dice Maluquer, que el panorama decimonónico sería de relativa estabilidad 6 . Pero el panorama de relativa estabilidad se transformó con el estallido de la I Guerra Mundial, ya que a partir de su inicio se generó una espiral inflacionista iniciada por el desplazamiento de la demanda exterior hacia productos que anteriormente se destinaban a cubrir la interior y por los aumentos de precios de materias primeras como consecuencia de las dificultades en la importación. Esto, aunque había provocado un aumento de los costes, también facilitó un aumento de los ingresos, de forma que habría aumentado la tasa de beneficios y, como reflejo, tales aumentos incentivaban las reivindicaciones obreras. Hay que añadir, además, una política fiscal que no quiso o no pudo «articular las medidas e instrumentos precisos para conseguir el control y la adecuada orientación de las rentas generadas durante la "coyuntura extraordinaria" de Guerra» ${ }^{7}$. En conjunto se produjo durante los primeros años del conflicto un desfase entre los precios y los salarios que comportó una caída considerable del salario real. Como señala Maluquer, no parece que este desfase fuese generalizado en todos los sectores ${ }^{8}$, pero por lo que podemos apreciar en el cuadro 3 sí que fue importante en el textil, aunque habría variado de empresa a empresa y según la zona. Las diferencias que se observan entre los índices de la provincia de Barcelona y el de Vilanova creo que hay que atribuirlas al hecho que, hasta 1913, el de Barcelona sólo incluye información de la Colonia Sedó y, a partir de 1913, en el índice se contabilizan informaciones procedentes de ésta junto con otras referentes a la industria lanera de Sabadell; existe, por tanto, la probabilidad de evoluciones diferentes.

Uno de los fenómenos que provocó la inflación durante el periodo de la I Guerra Mundial fue poner en el primer plano a los factores socio-políticos entre aquellos que marcan la evolución de los salarios, restando importancia a aquellos de carácter más estrictamente económico. De manera que el

\footnotetext{
6 Maluquer (1989), p. 504.

7 Roldán y Garcia Delgado (1973), p. 225.

${ }^{8}$ Maluquer (1989), pp. 507-508.
} 


\section{CUADRO 4}

Indices de salario real en la industria textil de la provincia de Barcelona y en Vilanova i la Geltrú $(1913=100)$

\begin{tabular}{|c|c|c|}
\hline & Barcelona & Vilanova \\
\hline 1909 & 91,6 & 112,9 \\
\hline 1910 & 95,2 & 124,8 \\
\hline 1911 & 95,7 & 118,5 \\
\hline 1912 & 99,1 & 104,8 \\
\hline 1913 & 100,0 & 100,0 \\
\hline 1914 & 101,0 & 105,8 \\
\hline 1915 & 98,2 & 104,9 \\
\hline 1916 & 89,4 & 96,2 \\
\hline 1917 & 92,1 & 93,3 \\
\hline 1918 & 91,8 & 87,6 \\
\hline 1919 & 84,8 & 89,2 \\
\hline 1920 & 124,1 & 113,1 \\
\hline 1921 & 129,1 & 139,2 \\
\hline 1922 & 139,5 & 165,6 \\
\hline 1923 & 136,4 & 159,2 \\
\hline $1924 \ldots \ldots \ldots \ldots \ldots \ldots \ldots$ & 131,4 & 140,1 \\
\hline 1925 & 131,4 & 136,8 \\
\hline
\end{tabular}

FUENTE: Para Barcelona Maluquer (1989), pp. 506-507. Para Vilanova la misma que el cuadro 1.

gran crecimiento del salario que se produce a partir de 1918 se explicaría en gran medida por la creciente fuerza del movimiento sindical. De la misma forma, la moderación del incremento salarial que se produce a partir de 1922 sería fruto de la presión que ejercieron las autoridades y el empresariado sobre dicho movimiento. Esto explicaría por qué el salario nominal masculino habría crecido durante el período bélico por encima de lo que lo había hecho el femenino, ya que el movimiento sindical contaba tradicionalmente con una menor presencia de las mujeres. Un fenómeno similar ha sido destacado por Enriqueta Camps para la segunda mitad del siglo XIX (hasta 1890) ${ }^{9}$.

Mientras el salario de los hombres fue siempre más elevado en la Fábrica de la Rambla que en la Colonia Sedó, la cosa no está tan clara cuando nos referimos al de las mujeres, siendo el salario de éstas o muy similar o, en algunas ocasiones, superior en Esparreguera que en Vilanova.

${ }^{9}$ Camps (1990), p. 202. 


\section{GRÁFICO 1}

Salarios nominales en la agricultura y en la industria en el Penedès

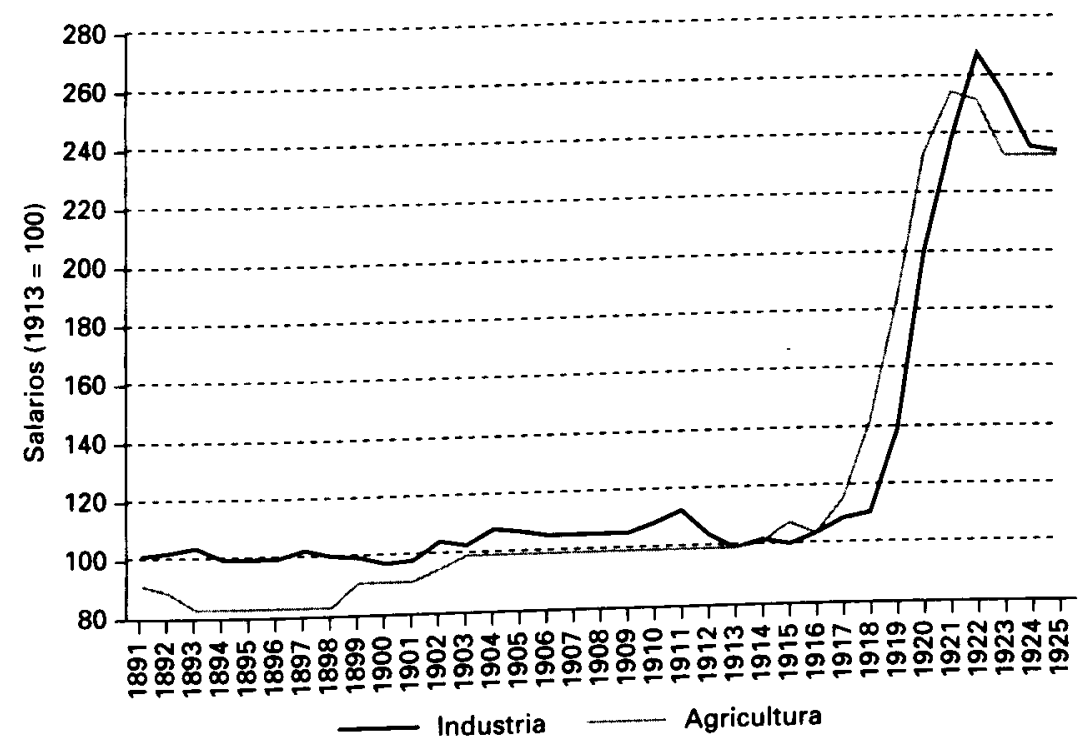

FUENTE: Para el salario industrial, las mismas del cuadro 1. Para el salario agrícola, Ramón Garrabou, Josep Pujol y Josep Colomé (1991), pp. 40-42.

\section{CUADRO 5}

Salarios nominales masculinos en la Colonia Sedó $y$ en la Fábrica de la Rambla (en pesetas semanales)

\begin{tabular}{|c|c|c|c|c|}
\hline & \multicolumn{2}{|c|}{ Hombres } & \multicolumn{2}{|c|}{ Mujeres } \\
\hline & $\begin{array}{l}\text { Colonia } \\
\text { Sedó }\end{array}$ & $\begin{array}{c}\text { Fábrica } \\
\text { de la Rambla }\end{array}$ & $\begin{array}{c}\text { Colonia } \\
\text { Sedó }\end{array}$ & $\begin{array}{c}\text { Fábrica } \\
\text { de la Rambla }\end{array}$ \\
\hline 1901 & 15,1 & 19,23 & 11,4 & 13,28 \\
\hline 1904 & 15,3 & 21,47 & 10,3 & 13,99 \\
\hline 1910 & 16 & 23,94 & 11,9 & 15,58 \\
\hline 1914 & 17,8 & 20,56 & 14 & 11,70 \\
\hline 1915 & - & 19,83 & - & 12,98 \\
\hline 1916 & 17,3 & 21,35 & 14,4 & 13,24 \\
\hline 1919 & 22,7 & 28,30 & 18,3 & 16,10 \\
\hline
\end{tabular}

FUENTE: Para la Colonia Sedó, Roser Nicolau (1983); para Vilanova, la misma que el cuadro 1. 


\section{CONCLUSIONES}

En definitiva, como se ha podido apreciar, podemos afirmar que todos los indicios apuntan a una evolución del salario en la industria textil, en el período 1890-1913, que vendría determinada por factores económicos. Junto a la productividad, la evolución de los beneficios y de las ventas, otro de los elementos que habría incidido fuertemente en aquella evolución sería la existencia de un mercado laboral próximo al núcleo industrial de Barcelona, en una ciudad donde también había de competir con otras industrias en la captación de los trabajadores y situada en una comarca en la que la agricultura exigía un contingente relativamente importante de mano de obra adulta masculina. Para la industria textil de la zona habría sido relativamente más fácil captar mano de obra femenina y menor de edad. Esto fue posible gracias a la existencia de una amplia capa de familias que tenían que completar sus ingresos empleando a las hijas o a los hijos no primogénitos fuera de la explotación familiar. Este sería el caso, mayoritariamente, de las familias «rabassaires», pero también de las familias de cuberos y marineros, cuyos ingresos no serían suficientes para cubrir la reproducción familiar ${ }^{10}$.

Este esquema de funcionamiento habría permitido moderar la tendencia al alza de los salarios y adecuarlos a las dificultades por las que hubiese pasado la empresa. Esto se quebró como consecuencia de la espiral inflacionista provocada por la I Guerra Mundial. El desfase entre precios y salarios que se produjo en la industria textil catalana habría alimentado el crecimiento del movimiento sindical, el cual había iniciado su reorganización a principios de la segunda década del siglo XX. A partir de 1914, los factores de tipo socio-político habrían pesado cada vez más en la evolución salarial, restando importancia a otro tipo de elementos que inciden en ella. Entre 1918 y 1921 el salario aumentó por encima de lo que lo hacían los precios y como reacción a la existencia de beneficios extraordinarios (en términos nominales). De la misma forma, la moderación del incremento salarial se explica por la presión empresarial y del Estado sobre el movimiento sindical

10 En este sentido, son interesantes los trabajos de Colomé (1996) y de García (1996). El primero analiza el papel que jugó la pequeña explotación campesina en el proceso de desarrollo de la viticultura catalana, centrándose en el caso de la comarca del Penedès y en el cual destaca la existencia de esta amplia capa de pequeños campesinos que necesitan colocar parte de la mano de obra familiar —en la industria y en el servicio doméstico, principalmente- para completar el ingreso. El segundo, que se ha centrado en el análisis de la formación del mercado de trabajo en varias ciudades industriales durante el primer periodo de la industrialización, vendría a corroborar, en el caso de Vilanova, las tesis expuestas por Colomé. 
una vez desaparecidos los factores que permitían la realización de beneficios, incluso antes de la Dictadura de Primo de Rivera.

Es evidente que en torno a la evolución de los salarios y de los precios en el período que abarca los inicios de la industrialización hasta la Guerra Civil de 1936 aún hay mucho que hacer. Creo que uno de los trabajos que quedan pendientes a partir de la presente nota es un análisis de la evolución de la Fábrica de la Rambla (trabajo iniciado ya por Jordi Nadal y Enric Ribas) que permita relacionar salarios, beneficios, rendimientos del capital, costes, productividad, que nos permitan ampliar el conocimiento que tenemos sobre el comportamiento histórico de la industria textil catalana, cosa que se verá facilitada por la extensa documentación conservada de la empresa aquí analizada.

\section{BIBLIOGRAFIA}

AA. VV. (1990): Simposi d'Anàlisi Econòmica, Secció d'Història Econòmica, vol. II, Universitat Autònoma de Barcelona.

CAMPS I CURA, Enriqueta (1990): «La evolución del salario real en el sector textil algodonero. La "España Industrial, S. A.", 1850-1913», Simposi d'Analisi Econòmica, Secció d'Història Econòmica, Universitat Autònoma de Barcelona, vol. II, pp. 197-205.

COLOMÉ, Josep (1996): L'especialització vitícola a la Catalunya del segle XIX. La comarca del Penedès, Tesis doctoral, Universidad de Barcelona.

GARCIA, Albert (1996): «Linici de la industrialització: els oficis», comunicación presentada al II Seminari d'Història del Penedès, El Vendrell.

GARRABOU, Ramón; PUjOL, Josep; COLOMÉ, Josep (1991): «Salaris, ús i explotació de la força de treball (Catalunya, 1818-1936)», Recerques, 24, Barcelona, pp. 23-51.

MALUQUER DE MOTES, Jordi (1989): «Precios, salarios y beneficios. La distribución funcional de la renta», en Albert Carreras (coord.): Estadisticas históricas de España, Fundación Banco Exterior, Madrid, pp. 496-532.

NADAL, Jordi (1992): Moler, tejer y fundir. Estudios de bistoria industrial, Ariel, Barcelona, pp. 211-239.

NICOLAU I NOS, Roser (1983): Trabajo asalariado, formación y construcción de la familia: la demanda de trabajo de la colonia textil Sedó y los comportamientos demográficos de la población, Tesis de licenciatura inédita, Bellaterra, pp. 89-92.

REHER, David S., y BALLESTEROS, Esmeralda (1993): «Precios y salarios en Castilla la Nueva: la construcción de un índice de salarios reales, 1501-1991», Revista de Historia Económica, Madrid, n." 1, pp. 101-149.

ROLDÁN, Santiago, y GARCfa DELGADO, José Luis (1973): La formación de la sociedad capitalista en España. 1914-1920, vol. I, Confederación Española de Cajas de Ahorro, Madrid.

SCHOLLIERS, Peter (1989): Real wages in 19th and 20th Century Europe. Historical and Comparative Perspectives, Berg, New York/Oxford/Münich. 